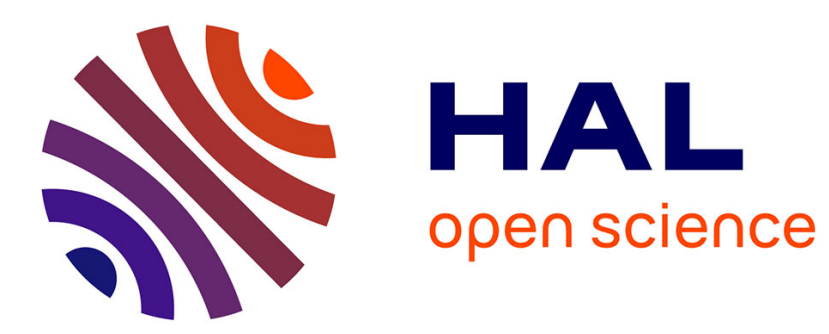

\title{
On ICA Based ICTD Classification of POLSAR DATA
}

Gabriel Vasile

\section{To cite this version:}

Gabriel Vasile. On ICA Based ICTD Classification of POLSAR DATA. IGARSS 2019 IEEE International Geoscience and Remote Sensing Symposium, Jul 2019, Yokohama, Japan. 10.1109/IGARSS.2019.8900558 . hal-02294724

\section{HAL Id: hal-02294724 \\ https://hal.science/hal-02294724}

Submitted on 23 Sep 2019

HAL is a multi-disciplinary open access archive for the deposit and dissemination of scientific research documents, whether they are published or not. The documents may come from teaching and research institutions in France or abroad, or from public or private research centers.
L'archive ouverte pluridisciplinaire HAL, est destinée au dépôt et à la diffusion de documents scientifiques de niveau recherche, publiés ou non, émanant des établissements d'enseignement et de recherche français ou étrangers, des laboratoires publics ou privés. 


\title{
ON ICA BASED ICTD CLASSIFICATION OF POLSAR DATA
}

\author{
Gabriel Vasile* \\ * Grenoble-Image-sPeach-Signal-Automatics Lab, GIPSA-lab \\ Univ. Grenoble Alpes, CNRS / Grenoble INP, Grenoble, France \\ gabriel.vasile@grenoble-inp.fr
}

\begin{abstract}
The Independent Component Analysis (ICA) has been recently introduced as a reliable alternative to identify canonical scattering mechanisms within PolSAR images. This paper addresses an important aspect for applying such methods on real data, namely statistical classification with ICA. A novel algorithm is proposed by adjusting the iterative segmentation from [1,2] to the particular nature of the Touzi's polarimetric decomposition [3]. This algorithm is tested using P-band airborne PolSAR data acquired for the ESA campaign TropiSAR campaign.
\end{abstract}

Index Terms - Independent Component Analysis, PolSAR, classification

\section{INTRODUCTION}

With Synthetic Aperture Radar (SAR) sensors being able to emit or receive two orthogonal polarizations, the Polarimetric target decompositions are PolSAR image interpretation techniques relying on the study of the interaction between the targeted area and the transmitted waveform $[4,5]$. In this context, the Incoherent Target Decomposition (ICTD) theory assumes that the scattering process is a combination of coherent speckle [6] and random vector scattering effects [7, 8].

In [9], a novel strategy to polarimetric ICTD was introduced by selecting the Independent Component Analysis (ICA) to identify the canonical scattering mechanisms within an image cell. The proposed ICA was able to retrieve nonorthogonal scatterer types [10, 11]. By applying the MMSE filter [12] on each of the ICA derived rotation invariant scattering vectors, we have shown in [13] that spatial resolution can be better preserved with respect to the conventional PolSAR boxcar speckle filter.

For example, the Cloude and Pottier $\mathrm{H} / \alpha$ feature space [14] is one of the most employed methods for unsupervised PolSAR data classification based on ICTD. The association of the coherence matrix eigenvectors to the most dominant scatters introduces unfeasible regions in the $\mathrm{H} / \alpha$ plane. It has been shown in [11] that the ICA provides additional information: unconstrained by the orthogonality condition between

Thanks to the French Aerospace Lab for providing the PolSAR data. the estimated scattering mechanisms that compose the PolSAR clutter under analysis, ICA is not subject to the unfeasible region in the $\mathrm{H} / \alpha$ plane, increasing the range of possible natural phenomenons depicted in this feature space.

The paper is structured as follows. Section 2 illustrates in several points the PolSAR classification algorithm proposed in the framework of the ICA ICTD, while Section 3 presents some qualitative and quantitative performance assessment. Section 4 concludes the paper.

\section{ICA-ICTD STATISTICAL CLASSIFICATION}

Polarimetric SAR images can be used for several applications, for example for land cover classification. The ICA based ICTD decomposition is based on the estimation of the mixing matrix $\mathbf{A}$ (Eq. 1). There are several criteria for determining the elements of $\mathbf{A}$ in order to ensure the mutual independence of the sources in $\mathbf{s}$. The common factor for all the applied methods is the assumption that at most one of the sources is Gaussian.

$$
\mathbf{k}^{c}(i, j)=\left[\begin{array}{lll}
A_{11}^{c} & A_{12}^{c} & A_{13}^{c} \\
A_{21}^{c} & A_{22}^{c} & A_{23}^{c} \\
A_{31}^{c} & A_{32}^{c} & A_{33}^{c}
\end{array}\right] \cdot\left[\begin{array}{l}
s_{1}^{c}(i, j) \\
s_{2}^{c}(i, j) \\
s_{3}^{c}(i, j)
\end{array}\right]=\mathbf{A}^{c} \mathbf{s}^{c}(i, j)
$$

The selected Complex Fast-ICA algorithm is based on a bottom-up approach: emphasizing the non-gaussanity of the sources by maximizing an arbitrary nonlinear contrast function (Eq. 2) whose extrema coincides with the independent component.

$$
J_{G}(\mathbf{w})=\mathbf{E}\left\{\mathbf{G}\left(\left|\mathbf{w}^{\mathbf{H}} \mathbf{x}\right|^{\mathbf{2}}\right)\right\}
$$

The performances of the algorithm depend strongly on the choice of the nonlinear function $G(y)$, which is supposed to be suited to the particular application. Therefore, here we have used the kurtosis criterion in deriving independent target vectors:

$$
G_{1}(y)=\frac{1}{2} y^{2} .
$$

In this case, the contrast functions becomes essentially a measure of the fourth statistical moment of the source. As its value in case of the Gaussian variable equals zero, by maximizing the kurtosis of each of the sources, we ensure their independence. 
The result of the incoherent target decomposition is the set of target vectors representing elementary scatterers and a set of scalars, providing their proportion in the total scattering. In our case, the target vectors of the independent scatterers are the columns of the estimated mixing matrix $\mathbf{A}=\mathbf{W}^{\mathbf{H}}$. The contribution to the total backscattering $(m)$ is computed as a square root of the maximal eigenvalue of the derived Graves matrix.

Being based on Kennaugh-Huynen condiagonalization projected onto the Pauli basis, the TSVM [3] allows parametrization of the target vector in terms of rotation angle $(\psi)$, maximum amplitude $(m)$, target helicity $\left(\tau_{m}\right)$, symmetric scattering type magnitude $\left(\alpha_{s}\right)$ and symmetric scattering type phase $\left(\Phi_{\alpha_{s}}\right)$, among which the last four are roll-invariant:

$$
\mathbf{k}=m|\mathbf{k}|_{m} e^{j \Phi_{s}}\left[\begin{array}{ccc}
1 & 0 & 0 \\
0 & \cos 2 \psi & -\sin 2 \psi \\
0 & \sin 2 \psi & \cos 2 \psi
\end{array}\right]\left[\begin{array}{c}
\cos \alpha_{s} \cos 2 \tau_{m} \\
\sin \alpha_{s} e^{j \Phi_{\alpha_{s}}} \\
-j \cos \alpha_{s} \sin 2 \tau_{m}
\end{array}\right]
$$

Using these parameters, it is eventually possible to represent the obtained independent target vectors on either symmetric or non-symmetric target Poincaré sphere. In our case, they do not necessarily form an orthogonal basis.

In this paper, we propose an extension of the statistical classification algorithm presented in [1] for direct application to ICA based ICTD.

One possibility would be to employ directly the mixing matrices from [9] and compute the local barycenter. However, the derived theory from [1] is valid with positive definite matrices, only. This does not hold for the ICA mixing matrices, which are not necessarily positive definite. The proposed solution consists in employing each ICA derived dominant scattering mechanism and form either three $3 \times 1$ or one $9 \times 1$ input complex random vectors.

The only restriction would be that the use of the ICA scattering mechanisms are not directly rotational invariant. As presented in [13], we propose once again to use the rotation invariant scattering vectors of the following form [3]:

$$
\overrightarrow{\mathbf{v}}^{\text {orient-inv }}=\mu\left[\begin{array}{c}
\cos \alpha_{s} \cos \left(2 \tau_{m}\right) \\
\sin \alpha_{s} e^{j \Phi_{\alpha_{s}}} \\
-j \cos \alpha_{s} \sin \left(2 \tau_{m}\right)
\end{array}\right]
$$

As described in [3], $\overrightarrow{\mathbf{v}}^{\text {orient-inv }}$ is obtained by constructing the Graves power matrix and performing the condiagonalization from [15] followed by the Huynen desying.

\section{POLSAR EXPERIMENTAL RESULTS}

The PolSAR dataset was acquired by the French Aerospace Lab (ONERA), in 2009, over the French Guiana, in the frame of the ESA campaign TropiSAR. Fig. 1 shows the initial and the LLMSE filtered span image in decibels.

After speckle filtering, the K-Means type iterative segmentation is applied using the Riemannian mean [2]. The maximum number of iterations has been set to $N=10$. Fig.
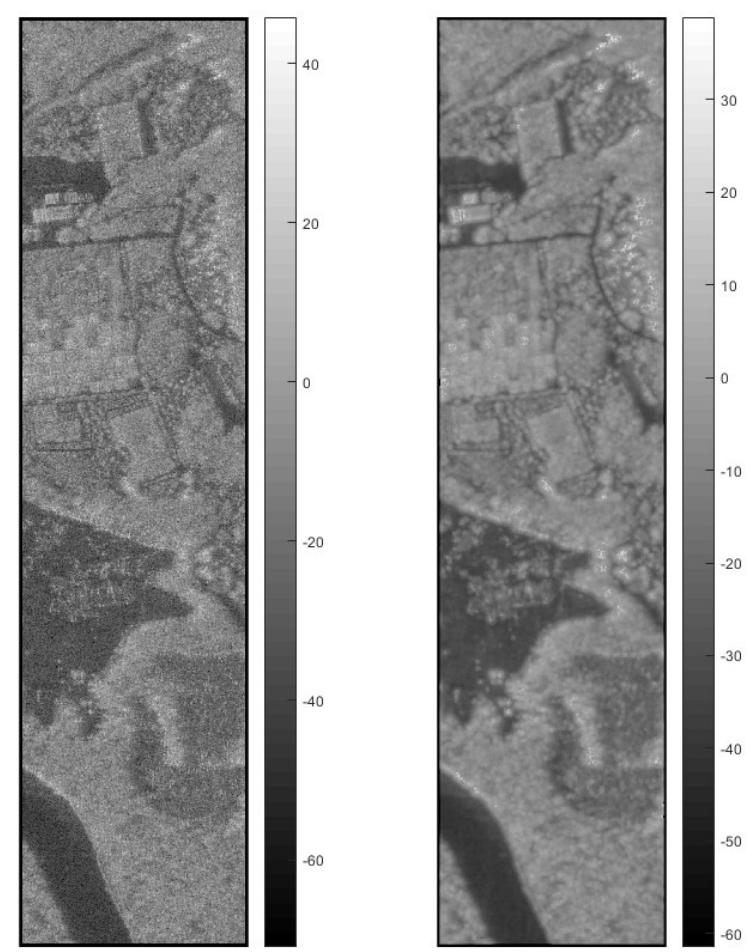

Fig. 1. Paracou P-band airborne dataset, span image in $d B$ : (left) initial and (right) filtered.

2 shows the rotation invariant scattering vectors in the Pauli basis corresponding to the first, second and third ICA scattering mechanisms.

Fig. 3 illustrates the Chernoff bond and the percentage of pixels moving to another class at each iteration. Both indicators are revealing that the optimal number of iteration for the proposed classification algorithm is 5 .

One can observe, in Fig. 4-(a),(b),(c), the barycenter evolution in the $\mathrm{H} / \alpha$ plane for the three ICA scattering mechanisms: the entropy is considerably decreasing with the number of iterations indicating a better statistical clustering, while the $\alpha$ angle is moving from anisotropic particle to dipole scattering (which corresponds to forested areas at $\mathrm{P}$ band).

Finally, the final statistical classification result is presented in Fig. 5.

\section{CONCLUSION}

This paper presented an iterative statistical classification algorithm particularly adapted to PolSAR data processing when the ICA ICTD is applied. It has been tested using P band airborne PolSAR data over forested areas.

At this stage, further developments are required in order to evolve the proposed algorithm towards an user friendly more intuitive application. 

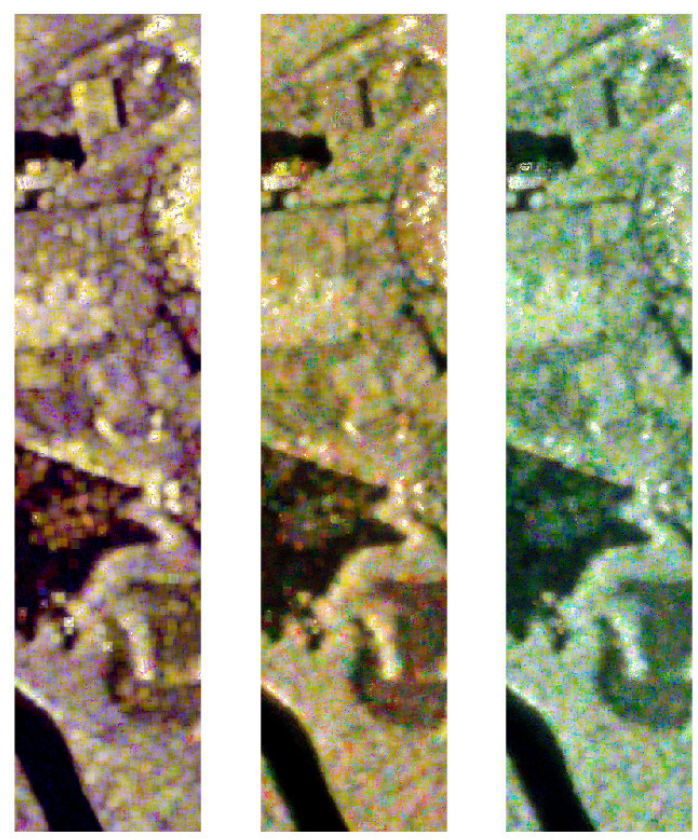

Fig. 2. Paracou P-band airborne dataset, Pauli color composition in $\mathrm{dB}$ : (left) first component, (middle) second component, (right) third component.

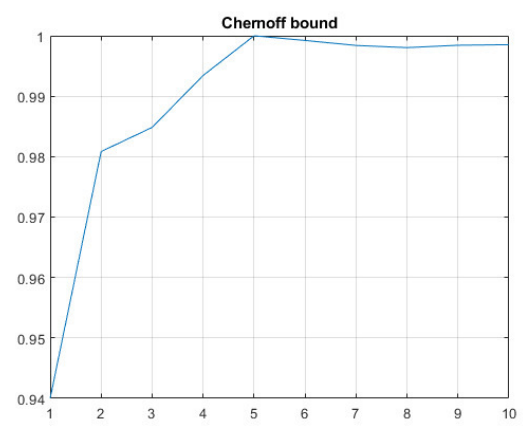

(a)

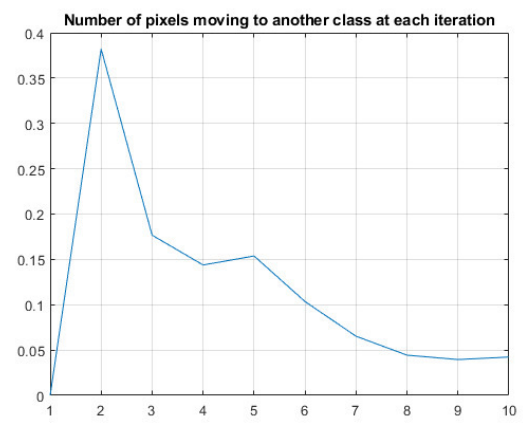

(b)

Fig. 3. Paracou P-band airborne dataset: (a) Chernoff bound and (b) percentage of pixels moving to another class at each iteration.

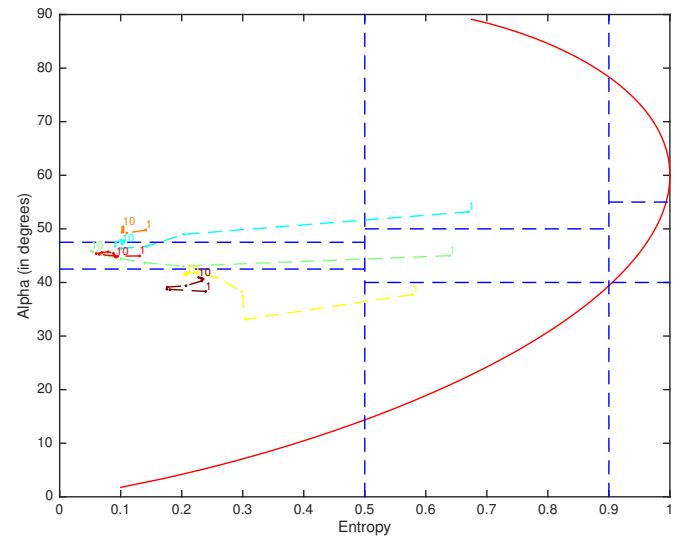

(a)

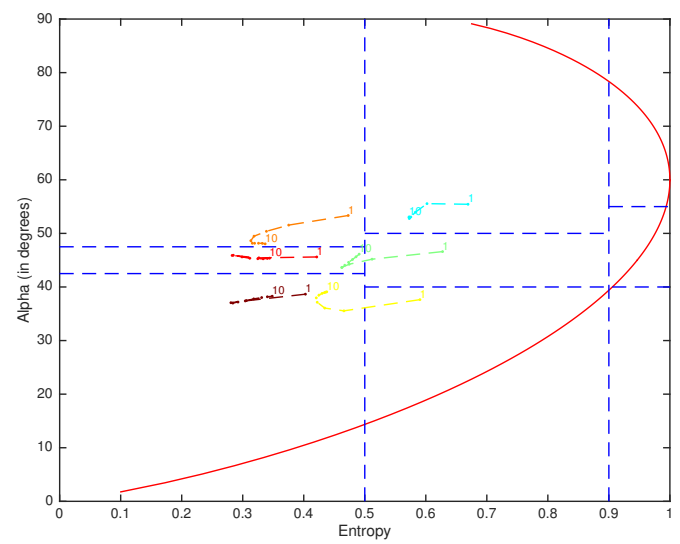

(b)

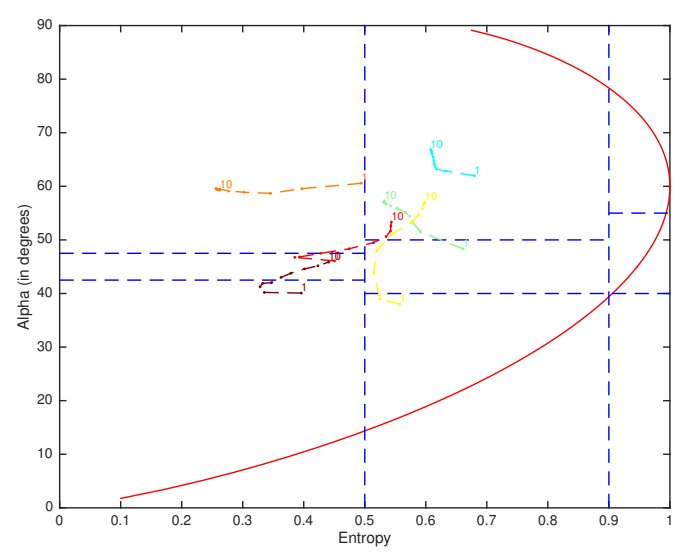

(c)

Fig. 4. Paracou P-band airborne dataset: $H / \alpha$ plane moving centers: (a) first component, (b) second component, (c) third component. 


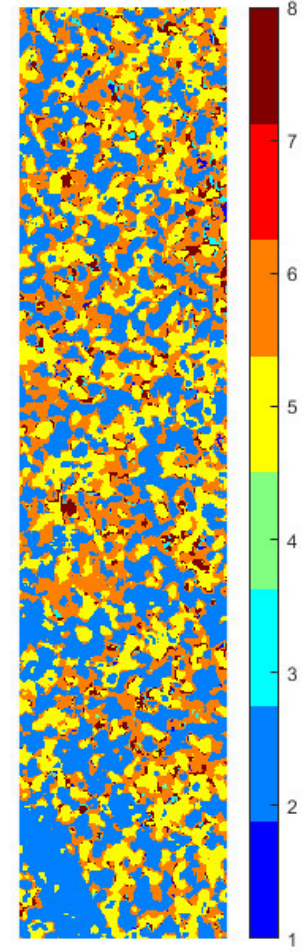

Fig. 5. Paracou P-band airborne dataset: (a) statistical classification map and (b) number of pixels moving to another class at each iteration.

\section{REFERENCES}

[1] P. Formont, F. Pascal, G. Vasile, J.-P. Ovarlez, and L. Ferro-Famil, "Statistical classification for heterogeneous polarimetric SAR images," IEEE Journal of Selected Topics in Signal Processing, vol. 5, no. 3, pp. 398-407, 2011.

[2] P. Formont, J.-P. Ovarlez, F. Pascal, G. Vasile, and L. Ferro-Famil, "On the extension of the product model in POLSAR processing for unsupervised classification using information geometry of covariance matrices," in Proceedings of the IEEE International Geoscience and Remote Sensing Symposium, Vancouver, Canada, 2011, pp. 1361-1364.

[3] R. Touzi, "Target scattering decomposition in terms of roll-invariant target parameters," IEEE Transactions on Geoscience and Remote Sensing, vol. 45, no. 1, pp. 7384, 2007.

[4] G. Vasile, Imagerie radar à synthèse d'ouverture interférométrique et polarimétrique. Application au suivi des glaciers alpins, Ph.D. thesis, Université de Savoie, France, 2007.

[5] N. Besic, G. Vasile, J. Chanussot, S. Stankovic, J.P.
Dedieu, G. d'Urso, D. Boldo, and J.P. Ovarlez, "Dry snow backscattering sensitivity on density change for SWE estimation," in Proceedings of the IEEE International Geoscience and Remote Sensing Symposium, Munchen, Germany, 2012, pp. 4418-4421.

[6] G. Vasile, E. Trouvé, J. S. Lee, and V. Buzuloiu, "Intensity-driven-adaptive-neighborhood technique for POLSAR parameters estimation," in Proceedings of the IEEE International Geoscience and Remote Sensing Symposium, Seoul, Korea, 2005, vol. 8, pp. 5509-5512.

[7] S. R. Cloude and E. Pottier, "A review of target decomposition theorems in radar polarimetry," IEEE Transactions on Geoscience and Remote Sensing, vol. 32, no. 6, pp. 498-518, 1996.

[8] R. Touzi, "Speckle effect on polarimetric target scattering decomposition of SAR imagery," Canadian Journal of Remote Sensing, vol. 33, no. 1, pp. 60-68, 2007.

[9] N. Besic, G. Vasile, J. Chanussot, and S. Stankovic, "Polarimetric incoherent target decomposition by means of independent component analysis," IEEE Transactions on Geoscience and Remote Sensing, vol. 53, no. 3, pp. 1236-1247, 2015.

[10] L. Pralon, G. Vasile, M. Dalla-Mura, J. Chanussot, and N. Besic, "Evaluation of ICA-based ICTD for PolSAR data analysis using a sliding window approach: convergence rate, gaussian sources, and spatial correlation," IEEE Transactions on Geoscience and Remote Sensing, vol. 54, no. 7, pp. 4262-4271, 2016.

[11] L. Pralon, G. Vasile, M. Dalla-Mura, and J. Chanussot, "Evaluation of the new information in the $\mathrm{H} / \alpha$ feature space provided by ICA in PolSAR data analysis," IEEE Transactions on Geoscience and Remote Sensing, vol. 55, no. 12, pp. 6893-6909, 2017.

[12] J. S. Lee, T. L. Ainsworth, Y. Wang, and K. S. Chen, "Polarimetric SAR speckle filtering and the extended sigma filter," IEEE Transactions on Geoscience and Remote Sensing, vol. 53, no. 3, pp. 1150-1160, 2015.

[13] G. Vasile, "Independent component analysis based incoherent target decompositions for polarimetric SAR data - practical aspects," in Proceedings of IEEE International Geoscience and Remote Sensing Symposium, Valencia, Spain, 2018, pp. 5859-5862.

[14] S. R. Cloude and E. Pottier, "An entropy based classification scheme for land applications of polarimetric SAR," IEEE Transactions on Geoscience and Remote Sensing, vol. 35, no. 1, pp. 68-78, 1997.

[15] A. Kostinski and W. M. Boerner, "On foundations of radar polarimetry," IEEE Transactions on Antennas and Propagation, vol. 34, no. 12, pp. 1395-1404, 1986. 